\title{
Descripción de patrones de apego en menores institucionalizados con problemas conductuales ${ }^{1}$
}

\section{Description of Attachment Patterns of Institutionalized Minors with Behavior Problems.}

\author{
Lorena Méndez Tapia* \\ Luis González Bravo**
}

\begin{abstract}
Resumen
La presente investigación evalúa patrones de apego en niños institucionalizados con problemas conductuales, utilizando criterios DSM- IV y la escala RADQ de Randolph, a través de un diseño de investigación alternativo a la "situación extraña de Ainsworth" y al AAI. Habiendo obtenido puntajes aceptables de validez y confiabilidad para el RADQ, se encontró que el 37,9\% de los menores identificados con problemas conductuales cumplían los criterios DSM-IV para trastorno reactivo de la vinculación y un 20,7 \% de la muestra cumplían los criterios diagnósticos de AD. Así mismo, el patrón de apego con más prevalencia corresponde al tipo ansioso, con un $38.3 \%$. Se revisan los alcances y limitaciones de estos hallazgos.
\end{abstract}

Palabras clave: apego, niños institucionalizados, problemas conductuales.

\begin{abstract}
The present investigation asesses attachment patterns in institutionalized children with conduct problems. In contrast, to previous designs which followed the Ainsworth Strange Situation or the AAI procedures, this investigation uses DSM - IV criteria and the Randolph Attachment Disorder Questionaire . After obtaining significant RADQ validity and confiability scores, it was found that $37,9 \%$ of the children with behaviour problems met the DSM -IV criteria for Reactive Attachment Disorder and 20,7\% met the diagnostic criteria for AD. Furthermore, the most frecuent attachment pattern was the Anxious type, (38,3\%). Finally, a review of the impact and limitations of the results is presented.
\end{abstract}

Key words: attachment, institutionalized children, behaviour problems

1 Esta investigación fue desarrollada gracias al financiamiento otorgado por la Dirección de Investigación de la Universidad San Sebastián, y no habría sido posible sin la colaboración de los centros que en esta participaron y de nuestros asistentes de investigación: Carolina Concha, Verónica Harris, Rosa Burguess, Andrés Sepúlveda, Paola Reyes, Katherine Eisele y Cecilia Sandoval.

* Docente, Escuela de Psicología, Universidad San Sebastían, Concepción. Chile. Email: Imendez@mater.uss.cl

**Docente Escuela de Psicología, Universidad San Sebastían, Concepción. Chile. 


\section{Introducción}

La gran mayoría de los niños que manifiestan conductas agresivas son diagnosticados con algún Trastorno asociado al Déficit Atencional y Comportamiento Perturbador. Sin embargo, estos criterios se limitan sólo a describir y no explicar las posibles causas de estos trastornos.

La intervención de los problemas conductuales en nuestro país consiste, en su mayoría, en el manejo de las conductas agresivas observables, aún cuando sabemos que existen otros factores asociados a la agresión y éstos no son tratados adecuadamente. Esto conlleva a la repetición de las conductas agresivas y al fracaso en cuanto al manejo preventivo de los comportamientos perturbadores.

Esta investigación pretende identificar y evaluar la psicopatología subyacente a la conducta agresiva y contribuir a una evaluación comprensiva de estos trastornos a partir de la teoría del vínculo afectivo.

Este enfoque tiene implicaciones terapéuticas ya que considera los factores subyacentes a la conducta perturbadora. Así, se pretende además plantear elementos a considerar en la intervención de los Trastornos del Comportamiento.

\section{Marco Teórico}

Según Bowlby $(1993,1995)$ el término apego se refiere a un sistema de conductas que son activadas por estrés y que tienen como objetivo la reducción del arousal y la reinstauración del sentido de seguridad, usualmente logrado en la infancia temprana a través de la proximidad física con un cuidador y más tarde dada por la disponibilidad psicológica de este. Según esta teoría, el sistema de apego motivacional consiste en cualquier forma de conducta que tiene como resultado el logro o la conser- vación de la proximidad con otro individuo claramente identificado que se considera mejor capacitado para enfrentar el mundo. Esto es obvio cuando la persona está asustada, fatigada o enferma, y se siente aliviada con el consuelo y los cuidados.

La investigación acerca del comportamiento de apego se ha desarrollado en dos grandes periodos. Desde 1970 a 1985, los estudios se centraron en la replicación de las investigaciones originales de Ainsworth y sus colegas en los cuales se observaba el comportamiento del infante hacia el cuidador, en respuesta a una situación extraña generada por la separación breve de la madre en un medio desconocido. El comportamiento observado ante el regreso de la madre se organizaba en tres patrones conductuales denominados: Seguro, Evitativo y Ambivalente (Ainsworht, Blehar, Waters, y Hall, 1978 citado en LyonsRuth, 1996). El infante seguro saluda a la madre a su regreso y se calma con el contacto físico y la aproximación afectiva de ésta. El niño seguro es capaz de restablecer el juego a nivel de pre-separación. El infante evitativo es menos tendiente a llorar ante la separación, no saluda a la madre a su regreso y la ignora inicialmente. La conducta evitativa tiende a aumentar del primer al segundo reencuentro. Los infantes ambivalentes (o resistentes) son propensos a llorar durante el episodio de separación y a menudo continúan llorando cuando la madre regresa. Son difíciles de consolar y muestran una aproximación pasiva. Desde 1985 al presente, la investigación, se concentró en estudiar la relación entre los tipos de apego y familias de bajo y alto riesgo. Los hallazgos indicaron que el comportamiento de algunos niños no coincidía con los patrones identificados previamente. Luego Main y Solomon (1990, en LyonsRuth, 1996) clasificaron una cuarta categoría denominada conducta de apego desorganizada. 
En la literatura mundial, la distribución de los patrones de apego en la población en general, hasta 1985, era de $65 \%$ seguro, $20 \%$ evitativo, y $14 \%$ ambivalente (van Ijzendoorn \& Kroonenberg, 1988 citado en Lyons-Ruth, 1996). En 1995, un estudio de meta-análisis, arrojó la siguiente distribución; $55 \%$ seguro, $23 \%$ evitativo, $8 \%$ ambivalente y $15 \%$ desorganizado (van Ijzendoorn, 1995 en Lyons - Ruth, 1996).

Un avance en la comprensión de las conductas de apego, fue dado por el desarrollo de la Entrevista de Apego Adulto (AAI) realizada por Main y Goldwyn en 1991 (Lyons-Ruth, 1996). Esta entrevista semiestructurada recoge las experiencias tempranas de apego del individuo y el significado que este le da actualmente a las experiencias pasadas de apego. El sistema de puntuación se basa en (1) descripciones de apego temprana (2) lenguaje utilizado en la entrevista (3) habilidad para entregar un relato integrado y creíble de experiencias de apego y su significado. Se considera que el lenguaje y el estilo del discurso corresponde a las representaciones de apego (George, Kaplan, \& Main, 1985, 1996 citado en Water y Hamilton, 2000)

Los individuos clasificados seguros son capaces de narrar sus historias coherentemente, valoran las relaciones de apego que perciben como significativas en su desarrollo. Son clasificados inseguros aquellos adultos cuyos relatos aparecen incoherentes. Los adultos clasificados indiferente (dismissive) niegan o minimizan el impacto de las relaciones de apego, tienen dificultad para recordar los eventos, a menudo idealizan las experiencias y describen antecedentes tempranos de rechazo. Los individuos en la categoría preocupado muestran confusión acerca de las experiencias tempranas, su descripción de la relación con los padres esta marcada por rabia o pasividad, e intentos mantenidos por complacer a los padres. La clasificación no resuelto se asigna si el relato del individuo acerca de eventos de vida (pérdida de un familiar o de una relación significativa, negligencia/abuso por parte de los padres o cuidador principal) es evaluado como incoherente (Main y Golwyn, 1985 - 1995 en Waters y Hamilton 2000).

\section{Interés clínico en niños institucionalizados}

Desde comienzos del siglo XX los clínicos se han interesado en el desarrollo psicológico y el bienestar de los niños institucionalizados (Chapin, 1915 en Zeanah, 1996). Durante los primeros cincuenta años, las objeciones hechas al cuidado institucional fueron minimizadas por la comunidad científica. Las observaciones de Skeel en 1966, se subestimaron debido a que los resultados implicaban que el medio tenía un impacto profundo en la inteligencia. Del mismo modo, muchas otras demostraciones acerca de la condición psicológica de los niños institucionalizados tuvieron poco impacto, quizás porque se estimaba que la condición psicológica era la causa y no el resultado de la crianza institucional. Algunos piensan que todas las publicaciones realizadas en las revistas profesionales no tuvieron el impacto, en términos de despertar consciencia, que tuvieron las películas de Rene Spitz $(1945,1946)$ acerca de niños institucionalizados (Zeanah, 1996)

Luego de la segunda guerra mundial, la Organización Mundial de la Salud (OMS) se interesa en evaluar las repercusiones para la salud mental en el caso de " niños que eran huérfanos o habían sido separados de sus familias por otras razones y precisaban de cuidado en familias adoptivas, instituciones $\mathrm{u}$ otros tipos de atención colectiva" (Bowlby, 1995). En su publicación El Cuidado Materno y Salud Mental, Bowlby resumió las observaciones de Spitz $(1945,1946)$ y de muchos otros acerca de los efectos dañinos 
de la institucionalización prolongada en el desarrollo de la personalidad. Según Bowlby, los niños que habían sido privados de sus madres, particularmente aquellos niños institucionalizados, sufrían en su desarrollo emocional, intelectual, verbal, social e incluso físico.

Rutter y cols., (2001) intentaron delinear los patrones conductuales de apego específicamente asociados a privación institucional. Un grupo de 165 niños rumanos adoptados antes de los 42 meses de edad fueron comparados a los 4 y 6 años de edad con 52 niños británicos no deprivados quienes habían sido adoptados durante la infancia. Los hallazgos mostraron que existe una asociación entre problemas de apego, desatención, hiperactividad, problemas pseudocognitivos y privación institucional. Sin embargo, no se encontró una asociación entre dificultades emocionales, problemas de relación con pares, problemas de conducta y deprivación institucional.

\section{Apego y Psicopatología}

En un estudio muy interesante, Fonagy y cols., (1996) investigaron la relación entre patrones de apego y clasificación psiquiátrica en una muestra de 82 internos no psicóticos y 85 casos control, utilizando la Entrevista de Apego para Adultos (AAI) y el sistema de clasificación de Main y Goldwyn (1991). Los pacientes psiquiátricos eran más propensos a clasificar dentro de la categoría preocupado y no resuelto con relación a la pérdida y el abuso. En el Eje I Ansiedad se asoció a la clasificación no resuelto mientras que en el Eje II, Trastorno de Personalidad Fronterizo fue asociado con trauma severo inconcluso.

Más específicamente los resultados indican que, las categorías preocupado y no resuelto son características de la población psiquiátrica. Este estudio proporcionó apoyo a la teoría de Bowlby validando la existencia de una asociación significativa entre psicopatología y relaciones conflictivas no resueltas de la infancia

Según Fonagy y sus colaboradores, la ansiedad indica el nivel al cual la experiencia traumática se vivencia en el presente, y se relaciona significativamente con apego no resuelto.

Por otro lado, se encontró que los pacientes con Trastorno Depresivo Mayor eran significativamente más propensos a ser seguros que los Bipolares o Distimicos (40\% vs. $19 \%$ y $10 \%$, respectivamente). Pacientes con Trastorno Bipolar tendían a clasificar más dentro de la categoría indiferente (38\%) que los pacientes con Trastorno Depresivo Mayor (7\%) o que los pacientes con Distimia (14\%)

Además, se encontró que los pacientes deprimidos tenían una visión más triste del mundo y menores expectativas de sus figuras de apego, lo cual explicaría la asociación entre depresión y una idealización disminuida de los padres.

La relación más específica demostrada en esta linea fue entre el diagnóstico de personalidad borderline y la clasificación de apego tipo preocupado. Un $88 \%$ de los pacientes borderline fueron clasificados como temerosos y preocupados por el trauma y la pérdida. Aún cuando este mismo autor señala que las diferentes clasificaciones de apego no se asocian a patologías específicas, en general la literatura relaciona estilos inseguros de apego con varias de ellas, siendo esta una fructífera y promisoria área de desarrollo para la psicología clínica (Jones, 1996). Según el autor, es difícil describir relaciones sistemáticas entre tipos de apego y tipos de clasificación clínica. Agrega que en general la clasificación diagnóstica depresión, no está exclusivamente conectada con la categoría no resuelto de apego adulto. Aún más, la relación 
entre trastorno oposicionista, trastorno disocial y apego tipo indiferente no es convincentemente clara, al igual que entre trastornos afectivos y el tipo de apego preocupado.

Rosenstein y Horowitz (1996, en Jones 1996), concluyeron que los adolescentes con una organización de apego tipo indiferente eran más propensos a presentar trastorno disocial, abuso de sustancias, personalidad narcisista o antisocial, y a presentar autoreportes de rasgos narcisistas antisociales y paranoides de personalidad. Los adolescentes con un tipo de apego preocupado eran más propensos a presentar un trastorno afectivo, obsesivo - compulsivo, histriónico, borderline o esquizotípico y autoreportaban rasgos de personalidad evitativo, ansioso, y distímico.

En otro estudio Cole-Detke \& Kobak (1996), evaluaron la relación entre estrategias de apego y el reporte de sintomatología depresiva y de trastornos de la alimentación, en un grupo de mujeres universitarias. Siguiendo la conceptualización de Main \& Weston (1990, 1982), Kobak y cols.,(1993 en Cole-Detke \& Kobak, 1996) reformularon el modelo de la teoría de control que asocia la apreciación del individuo acerca de la disponibilidad de la figura de apego con el desarrollo de estrategias de apego que regulan el despliegue de atención y la emoción. Desde esta perspectiva, una estrategia segura resulta del modelo de una figura disponible y sensible o un modelo del sí mismo como eficaz en elicitar y merecedor de una respuesta parental aún cuando exista una relación pobre con los padres. Esto permite al individuo desarrollar estrategias primarias para iniciar efectivamente conductas de apego y coordinar con flexibilidad las conductas de apego con otros sistemas de comportamiento. Las estrategias secundarias o defensivas se desarrollan cuando el individuo percibe las figuras de apego como insensibles y no disponibles. Según el mismo autor, la literatura de ape- go distingue dos tipos de estrategias secundarias. Un tipo de estrategia secundaria se desarrolla cuando el individuo percibe a la figura de apego como indiferente (ignoring) o rechazador de las señales de apego. Bajo esta situación el individuo desactiva el sistema de apego para reducir un potencial conflicto con la figura de apego. Esta desactivación se mantiene al desviar la atención dada a las señales de apego. Un segundo tipo de estrategia defensiva, se desarrolla cuando el individuo percibe la figura de apego como inconsistente, y para mantener la relación con el cuidador principal el individuo hiperactiva el sistema de apego con el fin de monitorear la figura de apego y maximizar el output. Los resultados de esta investigación indicaron que las mujeres con estrategias hiperactivadas eran más tendientes a reportar niveles más elevados de síntomas depresivos, mientras que las mujeres con estrategias desactivadas tendían a verbalizar índices más altos de sintomatología relacionada con trastornos de la alimentación.

\section{Apego y Déficit Atencional}

Para los fines de la presente investigación se centrará la atención en aquellos desórdenes considerados dentro del DSM-IV, en la categoría de Trastornos por Déficit de la Atención y Comportamiento Perturbador, que incluye Trastorno por Déficit de la Atención con Hiperactividad, Trastorno por Déficit de la Atención con Hiperactividad No especificado, Trastorno Disocial, Trastorno Negativista Desafiante, Trastorno de Comportamiento Perturbador No especificado.

Con relación específicamente al déficit atencional, se considera uno de los trastornos de salud mental más frecuentemente diagnosticado en USA, alcanzando entre el $2 \%$ y el $14 \%$ de los niños en edad escolar (Scahill y Schwab- Stone, 2000). En nuestro país no se han realizado estudios 
epidemiológicos comparables, aún cuando se ha encontrado una prevalencia de $6,2 \%$ en los niños de primero básico. (Bizama, 1998).

Así mismo se presenta en comorbilidad con otros trastornos disruptivos de la conducta, como el Trastorno Negativista Desafiante y el Trastorno Disocial (Biederman y col, 1993, citado en Scahill y SchwabStone, 2000), y a su vez, estos últimos trastornos ocurren con una tasa más alta a la esperada en la misma población escolar (Scahill y Schwab-Stone, 2000). Estos indicadores subrayan la necesidad de comprender la complejidad del fenómeno para diseñar adecuados programas de intervención.

En torno a la relación entre apego y déficit atencional, Yoshikawa (1997) realizó un estudio longitudinal con 40 infantes de 18 meses con características de comportamiento escurridizo manifestado en no mirar cuando eran llamados por los padres, ni tampoco confirmar o chequear la presencia de los padres. A los seis años de edad, 28 de estos niños fueron diagnosticados con Déficit Atencional con Hiperactividad, 6 resultaron con Retardo Mental y 4 con Trastorno Generalizado del Desarrollo..

La asociación entre la clasificación de apego inseguro en la infancia y futuros problemas conductuales ha sido mixta cuando los padres puntúan los problemas de conducta (Bates, Maslin, \& Frankel, 1985; Fagot \& Kavanaugh, 1990; Lewis y cols., 1984 en Zeanah, 1996). Esta asociación ha sido más consistente cuando los profesores u observadores puntúan problemas de conducta (Erickson y cols., 1985; Fagot \& Kavanaugh, 1990; Suess, Grossman \& Sroufe, 1992; Wartner, Grossman, Fremmer-Bombik, \& Suess, 1994 en Zeanah, 1996). Una investigación con una muestra de alto riego sugiere una fuerte asociación entre la clasificación de apego desorganizado y proble- mas conductuales en niños preescolares (Lyons-Ruth, Alpern \& Rapacholi, 1993).

\section{Apego y conducta agresiva}

Los trastronos de la conducta agresiva están definidos por dos categorías diagnósticas en el DSM-IV: Trastorno Negativista Desafiante (TND) y Trastorno Disocial (TDS). El diagnóstico de TND incluye conductas menos severas y menos antisociales que el TDS, tales como rehusar cumplir con las reglas de los adultos, pataletas, conducta provocativa, actitud desafiante, en cambio el diagnóstico de TDS requiere de comportamientos más agresivos o antisociales tales como, robo, provocar incendios o dañar la propiedad privada. Por lo tanto, niños agresivos menores de 6 años raramente son diagnosticados con TDS, pero la mayoría de los niños con TDS tienen historia de TND (Loeber, Lahey \& Thomas, 1991 en Lyons -Ruth, 1996). Estudios epidemiológicos de la población en general estiman que un $6 \%$ de los niños en edad escolar presentan TDS ( Robins, 1991 en Lyons-Ruth, 1996) y todos los estudios convergen concluyendo que en la edad escolar, el Trastorno Disocial es varias veces más frecuente en niños que en niñas con una incidencia de un $5 \%$ a $10 \%$ en niños y de $1 \%$ a $3 \%$ en niñas (Steward, Debois, Meardon, \& Cummings, 1986 en Lyons Ruth, 1996). En general, se estima que aproximadamente un $6 \%$ de los niños escolares presentan trastorno disocial (Robins, 1991).

Específicamente acerca de la relación entre apego y conducta agresiva, estudios longitudinales han encontrado una relación entre apego inseguro en la infancia- particularmente apego tipo evitativo - rabia y conducta desafiante hacia los padres y pares durante la infancia temprana y media y en los años preescolares (Ainsworth \& cols, 1978; Fagot \& Kavanagh, 1990; Matas, Arend, \& Sroufe, 1978; Erickson, Sroufe, \& Egeland, 1985 en Lyons-Ruth, 1996). Según 
el mismo autor, Main (1990) entrega una descripción de la estrategia subyacente al apego evitativo de los infantes, como restrictiva en la verbalización de rabia y malestar y desentendiéndose de las claves que podrían intensificar el deseo de buscar apoyo y confort. Otros estudios con familias más aventajadas económicamente, no han documentado con consistencia la relación especifica entre la estrategia de apego evitativo y el posterior comportamiento agresivo (Bates, Bayles, Bennett, Ridge, \& Brown, 1991; Fagot \& Kavanagh, 1990 en Lyons-Ruth, 1996)

Desde 1985, según Lyons - Ruth (1996) los estudios de apego comenzaron a centrarse en infantes con riesgo social o psicológico y codificar la presencia de apego desorganizado como también los tipos de apego ambivalente, evitativo y seguro. Estos estudios indican que el apego evitativo en familias de alto riesgo tiende a manifestarse de manera desorganizada en contraste a la forma organizada de apego evitativo en familias de infantes de bajo riesgo. Estudios más recientes han encontrado que es la desorganización, y no la evitación precisamente, lo que estaría asociado a la conducta agresiva. De acuerdo al mismo autor, estudios posteriores encontraron que los niños con apego seguro exhibían comportamiento prosocial hacia los padres $\mathrm{y}$ pares durante los años preescolares.

La investigación de apego indica que muchos de los correlatos del comportamiento agresivo infantil pueden ser identificados en la infancia, antes de que se inicien los ciclos de agresión. Es más, el apego desorganizado parece ser un precursor en el desarrollo futuro del comportamiento agresivo (Lyons-Ruth, 1996). Según el mismo autor, la clasificación de apego ambivalente es poco frecuente y no ha sido asociada con conducta agresiva.

En apoyo a esta línea de investigación, Greenberg, Speltz, \& DeKlyen (1993) en- contraron que los factores que contribuían a la etiología de la conducta agresiva estaban relacionados con la relación de apego, estresores familiares, disciplina y características del niño tales como temperamento o problemas neurobiológicos. Con relación a las conductas violentas, existe un esfuerzo importante por comprender las conductas agresivas en la infancia a partir de los desórdenes del apego (Myeroff \& cols, 1999).

En otro estudio, se examinaron los factores asociados a los problemas conductuales disruptivos iniciados en la infancia, en una muestra de 80 niños que habían sido derivados a una clínica psiquiátrica infantil y diagnosticados con trastorno negativista desafiante y un grupo control de 80 niños. Se estudiaron cuatro áreas: características de vulnerabilidad del niño, déficit en el estilo parental, apego inseguro y adversidad familiar. Los resultados mostraron que la combinación de estos cuatro factores proporcionaban alta sensibilidad, $81 \%$ y especificidad, $85 \%$, ( Rutter; 2001).

Otras investigaciones más recientes han utilizado clasificaciones, que aún cuando conservan algunos rasgos esenciales de las categorías tradicionales de Ainsworth o Main, incorporan algunos elementos novedosos y categorías originales. Este es el caso del trabajo desarrollado en el Centro de Apego de Evergreen, Colorado, el cual también será incorporado en la presente investigación, y que considera la categoría ansioso, la cual incluye a niños que habiendo tenido una historia de vinculación, esta fue disfuncional, y aparecen como sobre apegados e hiperdemandantes de afecto. Los niños evitativos, por otra parte, son más aislados y tienden a rechazar hostilmente manifestaciones de afecto o protección. En la categoría ambivalente, se incluyen menores que se caraterizan por su conducta desafiante y oposicionista, buscando el afecto con fines manipulativos. Los 
niños clasificados como desorganizados, son los más perturbados manifestando comportamiento bizarro y lábil. (Randolph, 2000)

\section{Metodología}

\section{Objetivo General:}

Determinar la relación existente entre patrones de apego y la presencia de trastornos conductuales en menores institucionalizados

\section{Objetivos Específicos:}

1.- Identificar menores institucionalizados diagnosticados con trastornos conductuales

2.- Evaluar patrones de apego en menores institucionalizados diagnosticados con comportamiento perturbador

3.- Correlacionar la prevalencia de los trastornos de comportamiento perturbador con patrones de apego

A. Población: Frente al hecho de que no existen antecedentes diagnósticos de la población de menores institucionalizados con trastornos del comportamiento perturbador, se considerará como población al total de niños institucionalizados en la Octava Región, el cual asciende a 8542 menores, de los cuales 3885 son mujeres y 4657 varones (SENAME, 2001).

B. Definición del tamaño estimativo de la muestra: La muestra a seleccionar fue de tipo No Probabilística e intencionada, ya que solo se evaluó a aquellos menores identificados y/o diagnosticados con problemas conductuales y que residen en la actualidad en centro de menores dependientes de la supervisión técnica del Servicio Nacional de Menores (SENAME).
Debido al hecho de que, en primer lugar, solo un bajo porcentaje de los menores institucionalizados disponen de un diagnóstico psicológico y/o psiquiátrico formal $\mathrm{y}$, en segundo lugar, en el caso de haberlo tenido en algún momento, no siempre se encuentra en el centro de atención, se utilizó un criterio amplio que permitía incluir tanto a aquellos menores con un diagnóstico psiquiátrico y/o psicológico que se incluya en la categoría de trastornos del comportamiento perturbador, como a aquellos que aún no teniéndolo formalmente, son reconocidos por sus cuidadores como manifestando problemas de conducta evidente.

Quedó finalmente una muestra de 58 sujetos extraída de los hogares Ciudad de Niño, Hogar Patricia Ortiz, CTD Nuevo Amanecer y Hogar Capullo

C. Definición de las variables:

1. Trastorno Reactivo de la Vinculación.

A. Definición Conceptual : Relación social que en la mayor parte de los contextos, se manifiesta marcadamente alterada e inapropiada para el nivel de desarrollo del sujeto, que se inicia antes de los 5 años de edad y que se asocia a una crianza sumamente patológica.(APA, 1994)

B. Definición Operacional : Cumplimiento de los criterios DSM-IV para trastorno reactivo de la vinculación.

2. Género.

A. Definición Conceptual : Forma que reciben las palabras para indicar el sexo (García-Pelayo, 1990: 500).

B. Definición Operacional : Respuesta dicotómica asignado en el espacio para sexo, y delimitado por las opciones hombre o mujer. 
3. Trastorno de apego

A. Definición Conceptual: Trastorno reactivo de la vinculación que se caracteriza tanto por cumplir los criterios de desorden reactivo de la vinculación del DSM-IV como los criterios del Desorden Negativista Desafiante. (Randolph, 2000).

B. Definición Operacional : Puntaje Obtenido en la Escala RADQ.

4. Trastorno de Conducta.

A. Definición Conceptual: Patrón de funcionamiento conductual caracterizado por un bajo nivel de adaptación a las normas e interacción social.

B. Definición Operacional: Valor numérico asignado en la categoría trastorno de conducta del protocolo de investigación.

D. Instrumentos Utilizados: Para el presente estudio se utilizaron 2 Instrumentos, los que a continuación de describen:

1.- Cuestionario de Desorden de Apego de Randolph (RADQ o Randolph Attachment Disorder Questionaire), adaptada y traducida por los autores, de Randolph (2000). Esta escala consta de treinta ítems con alternativa de respuesta tipo Likert de 1 a 5, que evalúa el constructo conocido como desorden de apego (AD). Este constructo es desarrollado principalmente por Elizabeth M. Randolph del Centro para el Tratamiento del Apego de Evergreen, USA.

La combinación de los distintos ítems en su sentido original (y algunos en un sentido inverso o r) permite identificar seis subescalas que discriminan los distintos sub-tipos de AD. Estas son AVD 1 (ítems 1, 2r, 3, 15, 16), ANX $1(5,10,17,22,23)$, AMB 1 (8r, 9r, 11r, 13r, 18r, 20r), AVD 2 (1, 3, 5r, 15, 16, 22r), ANX 2 (1r, 3r, 18, 20, 22), AMB2 (8r, 9r, 11r, 16r, 20r, 27r). Además incluye un procedimiento de análisis que permite descartar maltrato sin problemas de apego.

Se realizó una aplicación piloto para validar la prueba. Considerando que la mayoría de las investigaciones de apego en niños se han hecho en base al procedimiento conocido como Situación Extraña de Ainsworth, y que por lo tanto no se disponía de instrumentos psicométricos adecuados para ser utilizados como prueba criterio, se decidió utilizar dos instrumentos, que aún cuando no miden de forma específica y estandarizada el constructo original, consideran los autores que ilustra teóricamente lo que se entiende como vinculación.

La primera, el PSC o Pediatric Symptom Cheklist (Jellinek y cols., 1988) es una escala ampliamente utilizada para medir funcionamiento psicosocial y que cuenta con antecedentes de validez adecuados para nuestro país, con un alpha total de 0.853 (George y cols., 1994-1995). Para complementar este análisis se diseñó un instrumento que los autores denominaron Historieta Apego, y que consiste en un set de historietas que representan 5 situaciones diferentes de interacción entre adultos y niños. Cuatro de ellas representan patrones de apego alterados (ambivalente, desorganizado, evitativo, indiscriminado) y una que relataría una situación de apego normal entre un niño y algunos adultos.

Se le pidió a un grupo de 47 padres de niños de NSE similar a la que sería la muestra definitiva que contestara ambos instrumentos y el RADQ. A aquellos padres que seleccionaron historietas de apego alterado se le denominó con fines de análisis Grupo de Apego Anormal y a los que seleccionaron historietas de apego normal, se 
le denominó Grupo de Apego Normal.

Pues bien, las diferencias en las medias de los puntajes RADQ entre los grupos de Apego Anormal y de Apego Normal son significativas (con puntajes más altos para el grupo anormal), encontrándose un $t$ para varianzas distintas de 2.76 con un $\mathrm{p}<$ 0.001 . En el PSC también las diferencias son significativas encontrándose un t para varianzas distintas de 2.66 con un $\mathrm{p}<0.001$. Es resumen, las madres que tienden a calificar los patrones de apego de sus hijos como normales, tienden asimismo a reportar un mejor funcionamiento psicosocial y un puntaje más bajo en el RADQ. Esto ya es un hallazgo interesante.

El PSC arrojó una confiabilidad de 0.90 y el RADQ una confiabilidad de 0.92 . La correlación de Pearson entre el RADQ y el PSC fue de 0.811 con un $p$ $<0.001$.

2.- Protocolo de Investigación de Apego. Este protocolo de investigación fue diseñado exclusivamente para esta investigación y está basado en los criterios del Manual Diagnóstico y Psiquiátrico de Desórdenes Mentales de la Asociación Americana de Psiquiatría, DSM-IV (APA, 1994). Este protocolo se diseñó transformando cada uno de los cuatro criterios del DSM-IV para el Trastorno Reactivo de la Vinculación, en un procedimiento de recolección de datos.

Los Criterios del DSM IV para el trastorno reactivo de la vinculación de la infancia o la niñez son los siguientes:

A.- Relaciones sociales en la mayor parte de los contextos sumamente alteradas e inadecuadas para el nivel de desarrollo del sujeto, iniciándose antes de los cinco años de edad y puestas de manifiesto por (1) o (2):
(1) incapacidad persistente para iniciar la mayor parte de las interacciones sociales o responder a ellas de un modo apropiado al nivel de desarrollo, manifestada por respuestas excesivamente inhibidas, hipervigilantes, o sumamente ambivalentes y contradictorias.

(2) vínculos difusos manifestados por una sociabilidad indiscriminada con acusada incapacidad para manifestar vínculos selectivos apropiados.

B.- El trastorno del criterio A no se explica exclusivamente por un retraso del desarrollo (como en el retraso mental) y no cumple criterios de trastorno generalizado del desarrollo.

C.- La crianza patogénica se manifiesta al menos por una de las siguientes características:

(1) Desestimación permanente de las necesidades emocionales básicas del niño relacionadas con el bienestar, la estimulación y el afecto.

(2) Desestimación persistente de las necesidades físicas básicas del niño.

(3) Cambios repetidos de cuidadores primarios, lo que impide la formación de vínculos estables.

D.- Se supone que el tipo de crianza descrita en el Criterio $C$ es responsable del comportamiento alterado descrito en el criterio A. (por ej., las alteraciones del criterio A empezaron tras la instauración de los cuidados patogénicos que aparecen el Criterio C.)

Para el criterio A se diseñó una entrevista estructurada con 7 preguntas orientadas a evaluar lo considerado en el criterio. $\mathrm{Si}$ el adulto responde a cuatro de las siete preguntas en forma positiva, se considera presente el criterio. 
Para el criterio B, los evaluadores realizaron un diagnóstico diferencial según los criterios DSM-IV, descartando trastorno generalizado del desarrollo, retraso mental, Asperger y trastorno desintegrativo infantil.

Para el criterio $\mathrm{C}$ se revisó el expediente existente para cada niño en el Centro de Menores, identificando la ocurrencia en los dos primeros años de vida de los incidentes considerados en el criterio, utilizando para ello los informes judiciales, psicológicos, sociales, etc.

El criterio D fue confirmado utilizando las mismas fuentes.

Para los fines de la presente investigación se considerará como presencia de trastorno reactivo de la vinculación el cumplimiento de los cuatro criterios DSM-IV.

\section{Resultados}

A continuación se presentan los resultados de la aplicación de los instrumentos a la población estudiada.

Para comenzar se presentan los descriptivos de la muestra.

La muestra estuvo constituida por 58 menores, 18 mujeres y 40 varones. Las edades fluctuaron entre 6 y 16 años, siendo la media de 12 años y seis meses, y la desviación estándar de 2.46 años. A continuación se presentan las frecuencias de edad para la muestra total, y el detalle de la distribución por edad y género.
Tabla 1

\begin{tabular}{lcc}
\hline Edad & Frecuencia & Porcentaje \\
\hline 6 & 2 & 3.4 \\
8 & 2 & 3.4 \\
9 & 3 & 5.2 \\
10 & 6 & 10.3 \\
11 & 7 & 12.1 \\
12 & 4 & 6.9 \\
13 & 10 & 17.2 \\
14 & 12 & 20.7 \\
15 & 8 & 13.8 \\
16 & 4 & 6.9 \\
Total & 58 & $100 \%$ \\
\hline
\end{tabular}

Tabla 2

\begin{tabular}{lrrr}
\hline Edad & Mujer & Hombre & Total \\
\hline 6 & 1 & 1 & 2 \\
8 & & 2 & 2 \\
9 & 2 & 1 & 3 \\
10 & 4 & 2 & 6 \\
11 & 1 & 6 & 7 \\
12 & & 4 & 4 \\
13 & 2 & 8 & 10 \\
14 & 6 & 6 & 12 \\
15 & & 8 & 8 \\
16 & 2 & 2 & 4 \\
Total & 18 & 40 & 58 \\
\hline
\end{tabular}

Frente al hecho de que la mayor parte de los menores estudiados, como se ha descrito, no contaban en el centro con un diagnóstico psiquiátrico disponible, se creó un rótulo adicional a aquellos correspondientes a la categoría de trastornos del comportamiento perturbador del DSM IV. Así, la clasificación final incluye: Problemas Conductuales (01), Trastorno por Déficit de la Atención (02), Trastorno por Déficit de la Atención No Especificado (03), Trastorno Disocial (04), Trastorno Negativista Desafiante (05), Trastorno del Comportamiento Perturbador No Especificado(06). 
Tabla 3

\begin{tabular}{lc}
\hline Diagnóstico & Frecuencia \\
\hline Problemas Conductuales & 47 \\
Trastorno por Déficit de la Atención & 6 \\
Trastorno Disocial & 3 \\
Trastorno Negativista Desafiante & 2 \\
Total & 58 \\
\hline
\end{tabular}

Tabla 4

\begin{tabular}{lccc}
\hline $\begin{array}{l}\text { Tipo de Desorden } \\
\text { Conductual }\end{array}$ & Mujeres & Hombres & Total \\
\hline $\begin{array}{l}\text { Problema Conductual. } \\
\text { Déficit Atencional con }\end{array}$ & 16 & 31 & 47 \\
$\begin{array}{l}\text { Hiperactividad } \\
\text { Trastorno Disocial }\end{array}$ & 1 & 2 & 3 \\
$\begin{array}{l}\text { Trastorno Negativista } \\
\text { Desafiante }\end{array}$ & 1 & 1 & 2 \\
Total & 18 & 40 & 58 \\
\hline
\end{tabular}

A continuación se examinarán los resultados de la evaluación de la presencia del Trastorno Reactivo de la Vinculación (DSM IV) a través del instrumento Protocolo de Investigación de Apego.

De los 58 sujetos de la muestra original, el 37,9\% cumple los criterios DSM IV para Trastorno Reactivo de la Vinculación. En las tablas 5,6 y 7 se muestran estos resultados para el total de la muestra y distribuidos por género y edad.

Tabla 5

\begin{tabular}{lcc}
\hline Frecuencia & Porcentaje \\
\hline Cumple criterios Trastorno & & \\
Reactivo de la Vinculación & 22 & 37.9 \\
No cumple criterios Trastorno & & \\
Reactivo de la Vinculación. & 36 & 62.1 \\
& 58 & $100 \%$ \\
\hline
\end{tabular}

Tabla 6

\begin{tabular}{|c|c|c|c|c|c|}
\hline & Mujer & $\%$ & Hombre & $\% 7$ & Total \\
\hline $\begin{array}{l}\text { Cumple criterios } \\
\text { TRV }\end{array}$ & s 6 & 33.3 & 16 & 40 & 22 \\
\hline $\begin{array}{l}\text { No cumple } \\
\text { criterios TRV }\end{array}$ & 12 & 66.7 & 24 & 60 & 36 \\
\hline Total & 18 & 100 & 40 & 100 & 58 \\
\hline
\end{tabular}

Tabla 7

\begin{tabular}{lccc}
\hline Edad & $\begin{array}{c}\text { No cumple } \\
\text { TRV }\end{array}$ & $\begin{array}{c}\text { Si cumple } \\
\text { TRV }\end{array}$ & Total \\
\hline 6 & 2 & & 2 \\
8 & 1 & 1 & 2 \\
9 & 2 & 1 & 3 \\
10 & 3 & 3 & 6 \\
11 & 4 & 3 & 7 \\
12 & 3 & 1 & 4 \\
13 & 6 & 4 & 10 \\
14 & 7 & 5 & 12 \\
15 & 5 & 3 & 8 \\
16 & 3 & 1 & 4 \\
Total & 36 & 22 & 58 \\
\hline
\end{tabular}

$\mathrm{Al}$ analizar la distribución de los sujetos que califican para Trastorno Reactivo de la Vinculación, según el tipo de Trastorno Conductual que presenta, se obtienen los resultados ilustrados en el gráfico 1. Llama particularmente la atención que dentro de la categoría Problema Conductual, el $40.4 \%$ de los menores cumplan los criterios para Trastorno Reactivo de la Vinculación, según el DSM IV. 


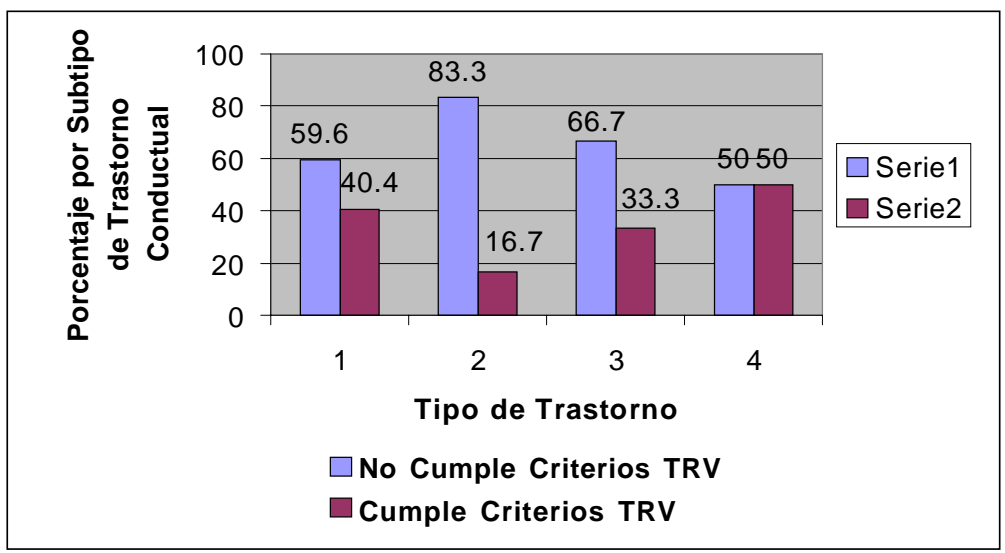

1.- Problema Conductual

2.-Déficit de la atención con hiperactividad

\section{3.- Trastorno Disocial \\ 4.- Trastorno Negativista desafiante.}

Los porcentajes descritos en el gráfico anterior deben ser analizados a la luz de la cantidad de sujetos incluidos en cada categoría. Por ejemplo, no es posible considerar como equivalentes el problema conductual presente en 47 sujetos con el trastorno negativista desafiante, que tiene sólo 2 sujetos.

Pasando a una tercera parte del análisis, se clasificó a los sujetos según el tipo de apego que presentaban. Para este propósito, se utilizaron las subescalas del RADQ que permiten situar a los sujetos en los distintos tipos de apego que la autora del instrumento identifica. Debemos recordar que la clasificación que Randolph utiliza no corresponde exactamente a la utilizada por Ainsworth y por otros autores a partir de la década de los 80. Randolph clasifica los tipos de apego en Evitativo, Ansioso, Ambivalente y Mixto, el cual representa una mezcla de algunos de los tipos anteriores.
De los 58 sujetos de la muestra inicial, sólo 47 pudieron ser sometidos a esta clasificación, principalmente por el hecho de que existían missing values que eran absolutamente necesarios para ella. Así no se pudo incluir el 19\% de la muestra original.

En este contexto, de los 47 menores evaluados, 6 cumplían los criterios de la categoría Evitativo (12.8\%), 18 los de la categoría Ansioso (38.3\%), 12 los de la categoría Ambivalente(25.5) y 11 los de la categoría mixto $(23.4 \%)$.

Así mismo, a través del RADQ se pudo identificar aquellos menores que cumplían los criterios diagnósticos para Desorden de Apego (AD, Attachment Disorder), la categoría utilizada por la autora del instrumento para describir los Trastornos de Vinculación, precisando que esta frecuencia ascendía a 12 , un $20.7 \%$ de la muestra total (58).

Además este instrumento permite identificar a aquellos menores que teniendo indicadores de haber experimentado maltrato, no cumplen los criterios para AD. En este caso estos suman 37 niños, con un 63.8 $\%$ de los casos.

Por otra parte, al identificar a aquellos menores que teniendo problemas de conducta, no cumplen los criterios de AD, se precisa que éstos suman 35, el $60.3 \%$ de la muestra. 
Al cruzar entonces la clasificación AD con la clasificación TRV nos encontramos con que sólo 7 de los 12 casos identificados positivamente como puntuando para $\mathrm{AD}$, cumplen también los criterios para TRV.

Existirían entre 8 y 9 casos (entre un $22.9 \%$ y un $24.3 \%$ ) que no cumpliendo los criterios de AD, si cumplen los criterios de TRV.
Por último, al analizar el tipo de vínculo inseguro según el tipo de desorden conductual que presenta (para un n válido de 47) y según género, se obtienen los resultados que se observan en la tabla 8 y en la tabla 9 .

Tabla 8

\begin{tabular}{lccccc}
\hline $\begin{array}{l}\text { Tipo } \\
\text { Vinculo }\end{array}$ & $\begin{array}{c}\text { Problema } \\
\text { Conductual }\end{array}$ & $\begin{array}{c}\text { Déficit } \\
\text { Atencional con } \\
\text { hiperactividad }\end{array}$ & $\begin{array}{c}\text { Trastorno } \\
\text { Disocial }\end{array}$ & $\begin{array}{c}\text { Trastorno } \\
\text { Oposicionista }\end{array}$ & Total. \\
\hline Evitativo & 5 & & 1 & & 6 \\
Ansioso & 12 & 4 & 1 & 1 & 18 \\
Ambivalente & 12 & 1 & & & 12 \\
Mixto & 10 & 5 & 2 & 1 & 11 \\
Total & 39 & & & & 47 \\
\hline
\end{tabular}

Tabla 9

\begin{tabular}{lrrr}
\hline Tipo Vínculo & Mujer & Hombre & \multicolumn{1}{c}{ Total } \\
\hline Evitativo & $2(13.3 \%)$ & $4(12.5 \%)$ & $6(12.8 \%)$ \\
Ansioso & $7(46.7)$ & $11(34.4 \%)$ & $18(38.3 \%)$ \\
Ambivalente & $2(13.3 \%)$ & $10(31.3 \%)$ & $12(25.5 \%)$ \\
Mixto & $4(26.7)$ & $7(21.9 \%)$ & $11(23.4 \%)$ \\
Total & $15(100 \%)$ & $32(100 \%)$ & $47(100 \%)$ \\
\hline
\end{tabular}

\section{Análisis y Discusión.}

Antes de proceder a analizar los resultados propiamente estadísticos, es necesario hacer algunas consideraciones. Sin duda, esta investigación no habría sido posible sin la total colaboración de las instituciones de menores con las cuales llevamos a cabo ésta. No obstante lo anterior, debemos señalar la gran cantidad de dificultades técnicas a las que nos vimos enfrentados al desarrollarla.
En primer lugar, sólo una mínima parte de los menores dispone en su carpeta de antecedentes de informes psiquiátricos y/ o psicológicos, que permitan avalar diagnósticos y planificar tratamientos en intervenciones con los menores. Entendemos que gran parte de esta situación se debe a la escasez de recursos humanos y materiales en el ámbito del sector público en nuestro país para realizar estas evaluaciones. Por otra parte, existe un bajo nivel de capacita- 
ción y sensibilización en el ámbito profesional acerca de la importancia de conocer en profundidad los diagnósticos DSM-IV, y de cuanto puede repercutir su uso adecuado en el trabajo psicosocial. Así, muchos de los menores que han sido evaluados o bien no disponen de un diagnóstico riguroso, o bien las categorías diagnósticas no corresponden a las categorías DSM- IV. ó CIE-10.

Esto se cruza profundamente con la realidad de los niños institucionalizados y de la atención de ellos en el sistema público. Los siempre insuficientes recursos económicos del sector público en este ámbito así como en el de la salud mental, sumado a la particular complejidad de la atención de estos menores, deja poco espacio para instancias de perfeccionamiento y coordinación intersectorial para los profesionales que en él trabajan. Los riesgos de esta situación, son perpetuar la cadena de situaciones vitales de exclusión y marginación que estos niños y niñas han venido enfrentado desde temprana edad. La importancia de un buen diagnóstico, además de uniformar criterios y precisar formulaciones o hipótesis explicativas para los fenómenos, es que permite distinguir la gravedad y el grado de rigidez e inmodificabilidad de ciertos cuadros.

La oportunidad de aprovechar resultados como los que presentamos en esta investigación, es factible sólo en un espacio de conversación donde es necesario que existan adecuados procesos de evaluación y diagnóstico que puedan ser revisados, criticados y modificados.

En particular en el caso del vínculo afectivo, el espíritu que mueve a los autores de la presente investigación es desplazar el foco de atención desde el típico diagnóstico conductual que rotula al niño con la supuesta e implícita convicción de que es muy difícil modificar "un mal comportamiento", hacia una evaluación de la calidad de las relaciones afectivas tempranas y actuales que el menor tiene, lo cual permitiría eventualmente (como sucede en países como EEUU y Canadá) diseñar planes de tratamientos coherentes con esta nueva visión.

En el caso nuestro, hemos constatado la dificultad de realizar investigaciones en torno al vínculo afectivo, además, por el hecho de que no se dispone de una variedad de instrumentos adecuados $y$ estandarizados a nuestra realidad, y que ofrezcan una alternativa real a procedimientos como la situación extraña de Ainsworth, para evaluar menores. Esto sucede con la AAI (Adult Attachment Interview) o el PBI (Parental Bonding Inventary), que más bien se aplican a poblaciones adultas.

Creemos que nuestra investigación ha hecho un aporte en sugerir al menos dos estrategias para evaluar patrones vinculares en niños. Primero, el instrumento creado como prueba criterio para el RADQ, las Historietas Apego, en la medida que se fundamentan en una aproximación visual más que lingüística, podría permitir acceder a las representaciones que los padres hacen con respecto al funcionamiento de sus hijos. Segundo, a pesar de las diferencias de los postulados de Randolph con los clásicos de Ainsworth, consideramos que nuestro estudio da a conocer en el ámbito nacional un instrumento de gran valor práctico como el RADQ. La pertinencia de los postulados básicos que están a la base de su construcción, a la luz de los hallazgos encontrados, serán discutidos luego.

Con respecto a la evaluación de los patrones de apego, recordemos, utilizamos dos fuentes primarias de información: los criterios DSM-IV para trastorno reactivo de la vinculación y los resultados del RADQ que permiten identificar AD.

Con respecto al primero, encontramos que un $37.9 \%$ de los menores cumplen los criterios de TRV. Esto es extremadamente 
interesante por varias razones. Primero, llama la atención que a pesar de las dificultades técnicas en el acceso de la información, en la existencia o no de ésta, en el tiempo que los cuidadores conocían a los niños, en que mucha de la información requerida requiere un análisis retrospectivo, etc., se hubiese encontrado esta prevalencia. Segundo, que ésta sienta las bases para estudios epidemiológicos que según el DSM- IV son limitados y desde donde se asevera que el TRV "parece ser muy poco frecuente". Tercero, existiría un evidente sub-diagnóstico de TRV en una población que por definición, está más expuesta a las situaciones de deprivación afectiva que están a la base de la etiología del TRV. Por lo menos, se puede afirmar que hay una desatención, por parte de los clínicos a aquellos signos que lo evidencian o, en general, a la dimensión explicativa de los fenómenos que considera como clave para iluminar conceptualmente las conductas del niño, las relaciones con la madre o los cuidadores primarios antes de los cinco años de vida. Cuarto, que el hecho de que se tome conciencia de los porcentajes aludidos incide profundamente en el diseño de planes de tratamiento para los Trastornos Conductuales en los niños, ya que se reposiciona como estrategia fundamental para la reparación el establecimiento de relaciones afectivas sólidas y de confianza.

Con respecto a los tipos de vínculo encontrados como más prevalentes en la población estudiada, se encontró el tipo ansioso con un $18 \%$. Esto no coincide con lo señalado con la literatura donde el más frecuente era el evitativo (van Ijzendorn, en Lyons-Ruth, 1996).

Antes de arribar a conclusiones apresuradas, es importante percatarse de que la categoría de tipo ansioso no pertenece a los tipos señalados por Ainsworth y otros autores, y que por lo tanto en una medida importante no es factible hacer comparaciones con los resultados de las investigaciones llevadas a cabo con anterioridad.

No obstante, el hecho de que la categorización aluda a un tipo de funcionamiento internalizante, tiene implicancias clínicas ya que muchos de estos menores identificados con problemas conductuales, probablemente presenten síntomas depresivos en la actualidad o en el corto plazo. Es decir, habría una gran cantidad de menores con un potencial de depresión importante que no estarían siendo visualizados y por supuesto, tampoco atendidos.

De hecho, las categorías precisadas por Randolph y el desarrollo de un constructo teórico como $\mathrm{AD}$, que además de incluir la conceptualización de vínculo más tradicional, incluye el Trastorno Negativista Desafiante, son aspectos que aún en el momento presente están sujetos a revisión y a una discusión teórica. Ya que el RADQ está construido sobre estas conceptualizaciones, aún debe ser analizado con una mirada crítica.

Sin embargo, en la medida que alcanzó buenos indicadores de confiabilidad y validez en nuestra aplicación piloto y a partir de la escasez de instrumentos en español que midan patrones de vinculación en niños y adolescentes, se considera que es una herramienta útil de conocer y aplicar.

Para terminar, recalcar la importancia de llevar a cabo más investigaciones como esta, que evidencien las necesidades afectivas y psicológicas de los niños deprivados e institucionalizados en nuestro país. 


\section{Bibliografía}

American Psychiatric Association (1994). Diagnostic and Statistical Manual of Mental Disorders, Fourth Edition. Washington, USA.

Bizama, I. (1998). Curso: Deficit Atencional en la Etapa Escolar. Servicio de Salud Concepción Arauco.

Bowlby, J. (1993). La separación afectiva. Editorial Paidós, Psicología Profunda. Primera impresión en español. Barcelona, España.

Bowlby, J. (1993). El vínculo afectivo. Editorial Paidós, Psicología Profunda. Primera impresión en español. Barcelona, España.

Bowlby, J. (1995). Una base segura. Aplicaciones clínicas de una teoría de apego. Editorial Paidós, Psicología Profunda. Primera reimpresión . Barcelona, España.

Cole-Detke, H. \& Kobak, R. (1996) Attachment processes in eating disorder and depression. Journal of consulting and clinical psychology. 64 (2).282-290.

Fonagy, P., Leigh, T., Steele, M., Steele, H., Kennedy, R., Matoon, G., Target, M. \& Gerber, A. (1996) The relation of attachment status, psychiatric classification, and response to psychoterapy. Journal of consulting and clinical psychology. 64 (1). 22-31.

Fonagy, P., TARget,. M. \& Gergely, G. (2000) Attachment and borderline personality disorder: a theory and some evidence. The Psychiatric Clinics of North America. 23 (1).103-122.

George, M., Siraqyan, X., Mores, R., Barra, F., Rodríguez, J., López, C. \& Toledo, V. (1994) Adaptación y validación de dos instrumentos de pesquisa de problemas de salud mental en escolares de $1^{\circ}$ básico. Revista Chilena de Psicología. (5), años 1994-1995.17-26.

GreenberG, M., T., Speltz.M.L., \& DeKlyen, M. (1993). The role of attachment in the early development of disruptive problems. Development and Psychopathology, (5), 191 - 213.

JellineK, M., Murphy, M., Robinson, J., Feins, A., LAmb, S. \& Fenton, T. (1988) Pediatric Symptom Checklist: Screening scool-age children for psychosocial dysfunction. The Journal of Pediatrics 112 (2), 201-209.

JONES, E. (1996) Introduction to the special section on attachment and psychopatology: part 1. Journal of consulting and clinical psychology. Vol. 64 (1).5-7.

Holland, R., Moretti, M., Verlaan, V. \& Peterson, S. (1993) Attachment and conduct disorder: the response program. Canadian Journal of Psychiatry. Vol. 38 (6).420-431.

Lyons-Ruth, K. (1996). Attachment relationsships among children with aggresive behavior problems: the role of disorganized erly attachment patterns. Journal of consulting and clinical psychology. 64 (1), 64-73.

Lyons-Ruth, K.,Alpern, L., \& Repacholi, B. (1993). Disorganized infant attachment classification and maternal psychosocial problems as predictors of hostile- agressive behavior in dthe preschool classroom. Child Development, $64,572-585$.

MAIN, M. (1996) Introduction to the special section on attachment and psychopatology: 2, Overview of the field of attachment. Journal of consulting and clinical psychology. Vol. 64 (2).237-243. 
Moretti, M., Holland, R. \& Peterson, S.(1994) Long term outcome of an attachment-based program for conduct disorder. Canadian Journal of Psychiatry. Vol. 39 (6). 360-371.

Mukaddes, N., Bilge, S., AlyanaK, B. \& Kora, M. (2000) Clinical characterístics and treatment responses in cases diagnosed as reactive attachment disorder. Child Psychiatry and Human Development. Vol. 30 (4). 273-287

Myeroff, R., Mertlich, G. \& Gross, J. (1999) Comparative effectiveness of holding therapy with agressive children. Child psychiatry and human develoment. Vol. 99 (4). 303-313.

RandolPh, E. (2000) Manual for the Randolph Attachment Disorder Questionaire. The Attachment Center Press,Tercera Edición. Evergreen, Colorado, USA.

Robins, L. (1991). Conduct Disorder. The journal of Child Psychology and Psiquiatry and Allied Disciplines.32, $193-212$.

Rosenstein, D. \& Horowitz H., (1996) Adolescent attachment y psychopatology. Journal of Consulting and Clinical Psychology. 64 (2), $244-253$.

RutTer, M. (1995) Clinical implications of attachment concepts, retrospect and prospect. Journal of Child Psychology and Psychiatry and Allied Disciplines. 36 (4). 549-571

Rutter, M., Kreppner J. \& O“Connor T. (2001). Specificity and heterogeneity in children's response to profund institutional privation. Developmental Psychology. Spring. 13 (2).97-103
Scanhill, L. \& Schwab-Stone, M. (2000) Epidemiology of attention- deficit/ hiperactivity disorder in scool-aged children. Child and adolescent psychiatrics clinics of north america. 9 (3). 541-555.

SENAME (1988) Estadísticas por región y sexo. Pagina Oficial del Servicio Nacional de Menores http://www.sename.cl/html/ estadisticas/region_sexo.pdf

Toth, S. \& CicchetTI, D. (1996) Patterns of relatedness, depressive symptomatology, and perceived competence in maltreated children. Journal of consulting and clinical psychology. 64 (1). 32-41

VAN IJZENDOORN, M. \& BaKermansKranenburG, M. (1996) Attachment representations in mothers, fathers, adolescents, and clinical groups: a metaanalytic search for normative data. Journal of consulting and clinical psychology. 64 (1).8-21.

Waters, E. \& Hamilton, E., (2000).The stability of Attachment Security from Infancy to Adolescence and Early Adulthood: General Introduction. Child Development. 71 (3). 678-683

Yoshikawa, R. (1997) Hyperactivity at 18 months of age and attention-deficict hiperactivity disorder before entry to school-follow up study from 18 months to 6 years. Seishin Shinkeigaku Zasshi. 99 (2). $47-66$

ZeAnah, C. (1996). Beyond insecurity: a reconceptualizacion of attachment disorders of infancy. Journal of consulting and clinical psychology. 64 (1).42-52

Período de límite de recepción de artículos: 30 de Marzo 2002.

Fecha de aceptación artículos: 30 de octubre 2002. 